\title{
Pulse Shaping for Differential Offset-QPSK
}

\author{
Andrew C. C. Lam, Akrum Elkhazin, Subbarayan Pasupathy, and Kostantinos N. Plataniotis
}

\begin{abstract}
Pulse shaping is examined as a means to improve the performance of a differential offset quadrature phase-shift keying system in a bandwidth-constrained environment. Through optimization with respect to a composite Nyquist criterion, the derived pulse shapes have comparable performance to a $\pi / 4$-differential quadrature phase-shift keying in an additive white Gaussian noise (AWGN) channel and better performance in a hard-limited AWGN channel.
\end{abstract}

Index Terms-Differential offset quadrature phase-shift keying (DOQPSK), pulse shaping.

\section{INTRODUCTION}

D IFFERENTIAL offset quadrature phase-shift keying (DOQPSK) is well suited to nonlinear bandlimited channels [1]. By limiting phase transitions between symbols to $\pi / 2$, DOQPSK has less out-of-band power than either differential quadrature phase-shift keying (DQPSK) or $\pi / 4$-DQPSK [2]. Furthermore, DOQPSK has less envelope fluctuation than $\pi / 4$-DQPSK, which leads to less spectral spreading if a nonlinear amplifier is used [3]. Since the in-phase (I) and quadrature-phase $(\mathrm{Q})$ symbols in DOQPSK are half a symbol apart, the differentially demodulated output generally contains intersymbol interference (ISI). If the transmitter and receiver pulse shaping filters obey the combined Nyquist 1 (N1) and Nyquist 2 (N2) criteria, ISI is limited to the previous and next symbols, and can be resolved by an eight-state Viterbi decoder [3]. The combined N1 and N2 criteria cannot be jointly satisfied for a pulse shape having a roll-off factor less than unity. This ISI can be mitigated by truncating the filter impulse response and applying a trellis decoder, whose complexity grows exponentially with the truncation length. Alternatively, multiple-bit differential detection has also been applied to DOQPSK [4], and reduced-complexity algorithms have been proposed [5].

In this letter, we consider a DOQPSK system with design roll-off factor of $\alpha=0.35$, and use pulse shaping as a means to improve performance without increasing complexity. Instead of expanding the number of trellis states, as in [2], to resolve ISI, we use a small trellis and examine how different pulse shapes can be used to minimize ISI. Using the closeness measure proposed in [6], different pulses are considered in terms of how closely they satisfy the N1 and N2 criteria. The pulse-shaping

Paper approved by G. M. Vitetta, the Editor for Equalization and Fading Channels of the IEEE Communications Society. Manuscript received July 23, 2005; revised December 7, 2005 and February 23, 2006. This paper was presented in part at the 22nd Biennial Symposium on Communications, Kingston, ON, Canada May 31-June 3, 2004.

A. C. C. Lam is with ATI Technologies Inc., Markham, ON L3T 7X6, Canada (e-mail: alam@ati.com).

A. Elkhazin, S. Pasupathy, and K. N. Plataniotis are with the Department of Electrical and Computer Engineering, University of Toronto, Toronto, ON M5S 3G4, Canada (e-mail: aelkhazi@comm.utoronto.ca; kostas@dsp.toronto. edu; pas@comm.utoronto.ca).

Digital Object Identifier 10.1109/TCOMM.2006.881349 strategy developed in this letter can improve BER performance with no increase in complexity.

\section{SySTEM MODEL}

We consider the DOQPSK in [2] and [3] that is shown in Fig. 1. At the transmitter, antipodally modulated bits are split into two bit streams $\left\{a_{k}, b_{k}\right\}$ that are differentially encoded into antipodal bit streams $\left\{x_{2 k}=a_{k} x_{2 k-2}, y_{2 k+1}=b_{k} y_{2 k-1}\right\}$, such that $x_{k}=0$ at odd intervals of $k$ and $y_{k}=0$ at even intervals of $k$. The quadrature-phase stream is delayed by $T_{s} / 2$, where $T_{s}$ is the symbol time, and both streams are passed through pulse-shaping filters and modulated. At the receiver, the received signal is demodulated, passed through a pulse-shaping matched filter, and sampled at intervals of $T_{s} / 2$. In discrete time, the received signal can be represented as

$$
r_{k}=\left[\sum_{i=-\infty}^{\infty} h_{i}\left(x_{k-i}+j y_{k-i}\right)\right] e^{j \phi_{k}}+n_{k}
$$

where $\left\{h_{i}\right\}$ is the impulse of the combined transmitter and receiver pulse-shaping filters, $\phi_{k}$ is the slowly time-varying phase, and $n_{k}$ is a noise source that is additive white Gaussian noise (AWGN), provided that the pulse shaping satisfies N1. Although N1 is not strictly satisfied for the candidate pulse, we assume that correlation between noise samples is negligible for the purpose of the receiver design.

The pulse-shaping filter is typically designed to satisfy the $\mathrm{N} 1$ criterion to eliminate ISI and the N2 criterion, to ensure accurate timing recovery. The unique minimum bandwidth pulse satisfying the combined $\mathrm{N} 1$ and $\mathrm{N} 2$ criteria is the full raised cosine (FRC) pulse that has $\alpha=1$ [7]. The N1 criterion implies $h_{1}=1$ and $h_{i}=0, i= \pm 2, \pm 4, \ldots$, in (1), and the N2 criterion implies $h_{ \pm 1}=(1 / 2)$ and $h_{i}=0, i= \pm 3, \pm 5, \ldots$ With the $\mathrm{N} 1$ and N2 criteria satisfied, the received signal is $r_{2 k}=$ $\left[x_{2 k}+j 0.5\left(y_{2 k-1}+y_{2 k+1}\right)\right] e^{j \phi}+n_{2 k}, r_{2 k+1}=\left[0.5\left(x_{2 k}+\right.\right.$ $\left.\left.x_{2 k+2}\right)+j y_{2 k+1}\right] e^{j \phi}+n_{2 k+1}$. Since the I and Q channel symbols are offset by $T_{s} / 2$, each symbol is interfered with by the previous and next symbols on the opposite channel.

The received signal is passed through a differential demodulator $\left(r_{k} r_{k-2}^{*}\right)$ in order to remove the unknown phase $\phi$. The demodulator output contains ISI that can be resolved using the Viterbi algorithm. Following the work in [2] and [3], a trellis can be constructed based on the noiseless demodulator outputs that are given by

$$
\begin{aligned}
\widehat{X}_{2 k} & =\Re\left(r_{2 k} r_{2 k-2}^{*}\right) \\
& =x_{2 k} x_{2 k-2}+\frac{1}{4}\left(y_{2 k-1}+y_{2 k+1}\right)\left(y_{2 k-3}+y_{2 k-1}\right) \\
\widehat{Y}_{2 k} & =\Im\left(r_{2 k} r_{2 k-2}^{*}\right) \\
& =\frac{1}{2} x_{2 k-2}\left(y_{2 k-1}+y_{2 k+1}\right)-\frac{1}{2} x_{2 k}\left(y_{2 k-3}+y_{2 k-1}\right)
\end{aligned}
$$




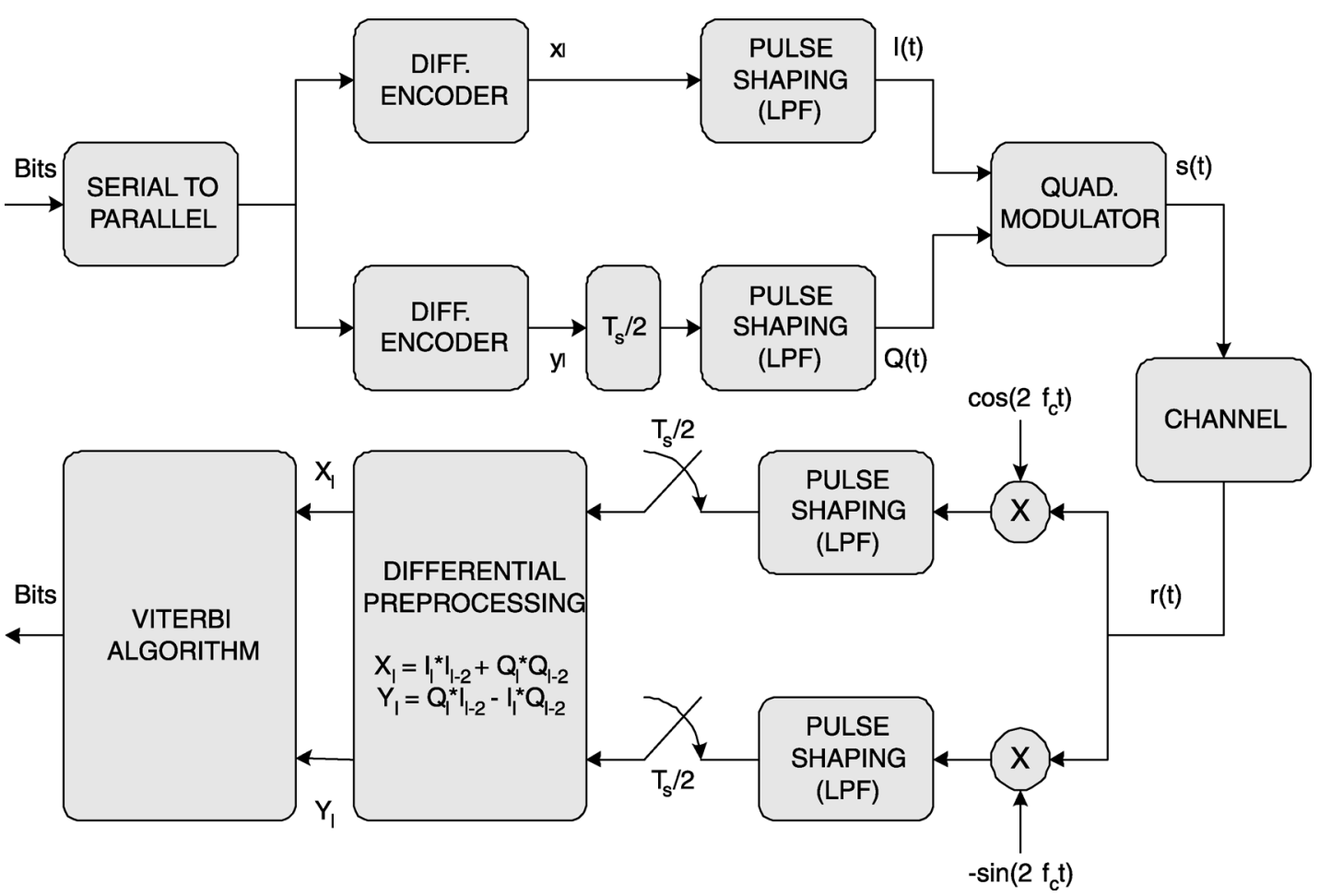

Fig. 1. System model.

$$
\begin{aligned}
\widehat{X}_{2 k+1}= & \Re\left(r_{2 k+1} r_{2 k-1}^{*}\right) \\
= & y_{2 k+1} y_{2 k-1}+\frac{1}{4}\left(x_{2 k}+x_{2 k+2}\right)\left(x_{2 k-2}+x_{2 k}\right) \\
\widehat{Y}_{2 k+1}= & \Im\left(r_{2 k+1} r_{2 k-1}^{*}\right) \\
= & -\frac{1}{2} y_{2 k-1}\left(x_{2 k}+x_{2 k+2}\right) \\
& +\frac{1}{2} y_{2 k+1}\left(x_{2 k-2}+x_{2 k}\right) .
\end{aligned}
$$

The noiseless output $\left\{\widehat{X}_{k}, \widehat{Y}_{k}\right\}$ is a function of four binary variables which yield a $2^{4}=16$-state trellis, which can be further reduced to an eight-state trellis by exploiting trellis symmetries [2], [3]. The branch metric computation is done according to $\Omega=\left(X_{k}-\widehat{X}_{k}\right)^{2}+\left(Y_{k}-\widehat{Y}_{k}\right)^{2}$, where $X_{l}$ and $Y_{l}$ are the real and imaginary parts of the differentially demodulated noisy received signal. Finally, differential decoding is performed according to $\hat{a}_{k}=x_{2 k} x_{2 k-2}$ and $\hat{b}_{k}=y_{2 k+1} y_{2 k-1}$ for the I and Q channels, respectively.

\section{DOQPSK FOR ROLL-OFF FACTORS LESS THAN 1}

The DOQPSK receiver described in the previous section has good performance and reasonable complexity roll-off factor if a unity roll-off pulse-shaping filter is used. For $\alpha=0.35$ roll-off factor, however, the N1 and N2 criteria cannot be mutually satisfied, resulting in interference from an infinite number of symbols, not simply the previous and next symbols on the opposite channel. The additional ISI introduced by bandwidth constraints can be accounted for by keeping more terms in the pulseshaping filter when forming the trellis state. This is the approach taken in [2]. If the filter $\left\{h_{i}\right\}$ is truncated after $l$ terms, the resulting trellis has $2^{2 l+1}$ states, thus, complexity grow exponentially with the number of terms in the filter.
As a low-complexity alternative to the trellis-state expansion in [2], we fix the trellis size and propose using pulse shaping to minimize ISI. Since there no pulse that satisfies the combined $\mathrm{N} 1$ and N2 criterion for $\alpha=0.35$, we strive to design pulses that minimize errors in both criteria. Employing the measure of closeness proposed in [6], the mean-squared error with respect to the $\mathrm{N} 1$ and $\mathrm{N} 2$ criteria is defined as

$$
\begin{aligned}
e_{I}^{2} & =\sum_{k}\left[h(k)-\delta_{k 0}\right]^{2} \\
e_{I I}^{2} & =\sum_{k}\left[h\left(\frac{2 k-1}{2}\right)-\frac{1}{2}\left(\delta_{k,-1}+\delta_{k 1}\right)\right]^{2}
\end{aligned}
$$

where $\delta_{i j}$ is the Kronecker delta. In (3) and (4), it has been assumed that $T_{s}=1$ for notational simplicity. The composite Nyquist error given by [6]

$$
e^{2}=\gamma e_{I}^{2}+\zeta e_{I I}^{2}
$$

where $\gamma$ and $\zeta$ are the weights given to the first and second criteria, respectively. A family of pulses can be derived by setting the parameters $\gamma, \zeta$ and optimizing the filter design with respect to the criterion in (5). Minimizing (5) for a particular $\gamma, \zeta$ yields an overall filter response given by [6], [8].

$$
H_{\gamma, \zeta}(f)= \begin{cases}\frac{\zeta \cos (\pi f)-\lambda}{\gamma+\zeta}, & 0 \leq|f| \leq \frac{1-\alpha}{2} \\ \frac{\gamma \cos (\pi f)-\lambda}{2 \gamma}, & \frac{1-\alpha}{2} \leq|f| \leq \frac{1+\alpha}{2} \\ 0, & \text { otherwise }\end{cases}
$$

where $\lambda$ is used to normalize the pulse, and is such that $\int_{-(1+\alpha) / 2}^{(1+\alpha) / 2} H_{\gamma, \zeta}(f) d f=1$. The rationale for choosing these measures of closeness and the additive composite error is twofold. First, the derivation becomes mathematically tractable, 
TABLE I

SUMMARY OF PULSE SHAPES

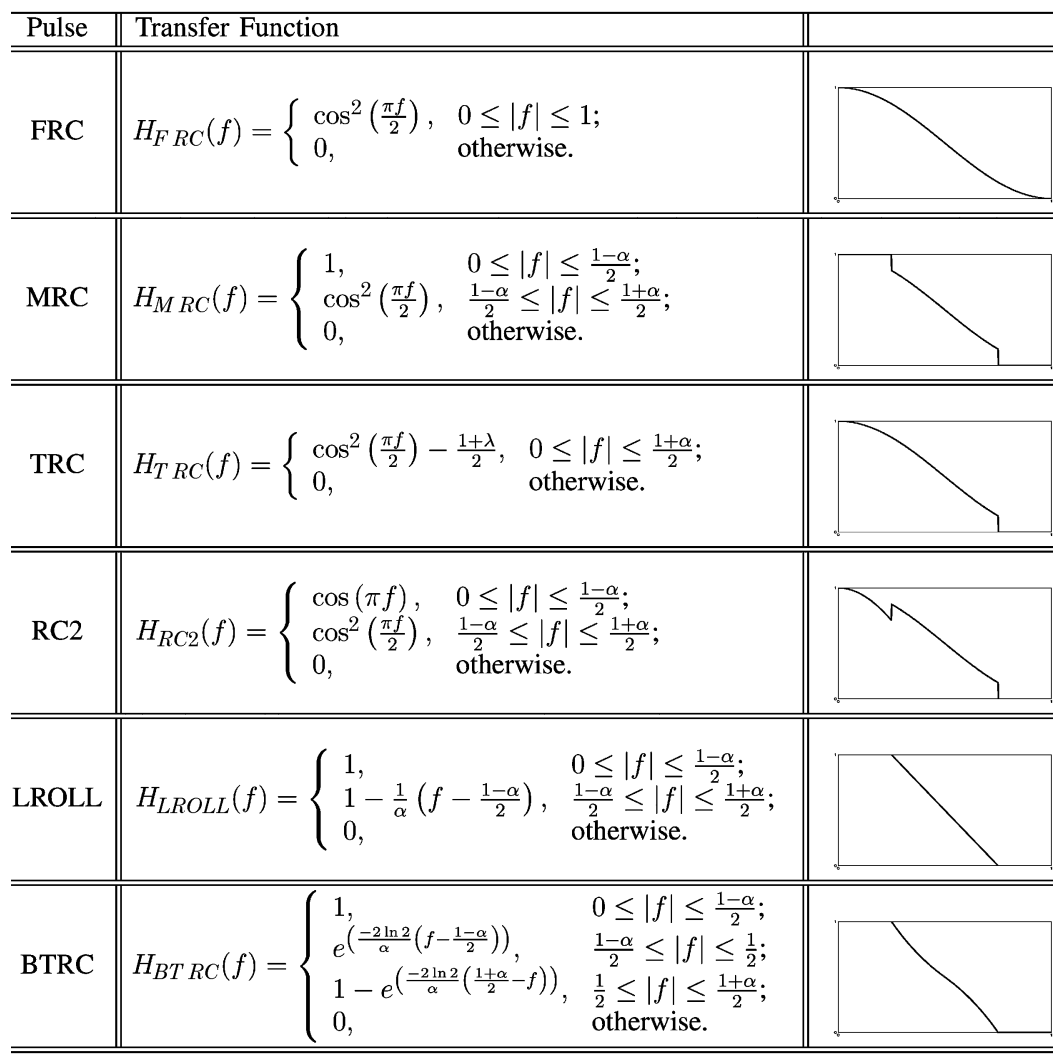

and second, the FRC pulse result for $\gamma=\zeta=1$ with a unity roll-off. We next derive three pulses by controlling $\gamma$ and $\zeta$.

1) Modified Raised Cosine: The modified raised cosine (MRC) (Table I) is a pulse that minimizes (4) subject to the N1 constraint. Thus, it satisfies $\mathrm{N} 1$, and is as close as one can get to N2. It is obtained by setting $\gamma$ and $\zeta$ such that $\gamma \gg \zeta$.

2) Truncated Raised Cosine: If $\gamma=\zeta=1$, the truncated raised cosine (TRC) (Table I) results. It simultaneously minimizes(3) and (4), but satisfies neither N1 nor N2. Since its area is dependent on $\alpha$, it has to be normalized by setting $-((1+$ $\lambda) / 2)=(1 /(1+\alpha))-(\sin \pi((1+\alpha) / 2) /(1+\alpha) \pi)-(1 / 2)$.

3) Raised Cosine 2: If we insist on keeping N2 at the expense of errors in N1, then the raised cosine 2 (RC2) (Table I) pulse results. In this case, the weights are set with $\zeta \gg \gamma$.

4) Continuous Filters: The three filters previously discussed have discontinuities in their spectra which can lead to difficulties in practical implementation. We consider two continuous spectral pulses both satisfying N1, namely: the linear rolloff (LROLL) [6] and the better than raised cosine (BTRC) [9] pulses (Table I). These pulses are not derived with respect to composite error criteria (5), although they have errors in N2 that are comparable to the MRC and TRC pulses.

\section{Simulation Results}

In this section, the effectiveness of the different pulse-shaping filters in a DOQPSK transmission is examined, with the $\pi / 4$-DQPSK and 128-state DOQPSK [2], [3] systems used as a reference. We consider the AWGN channel and the hard-limited

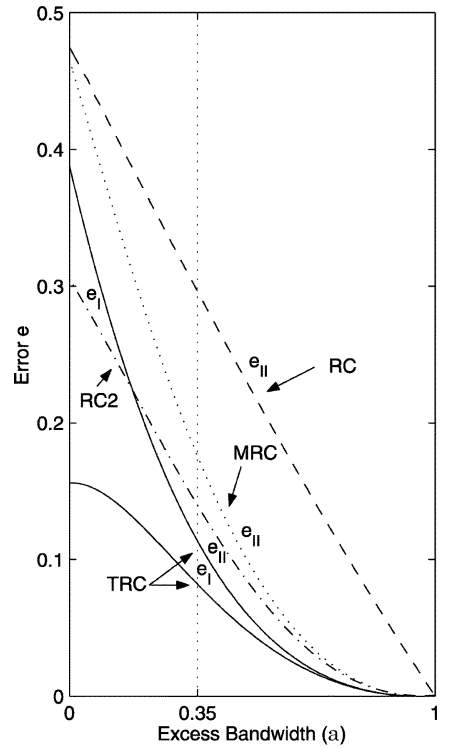

(a)

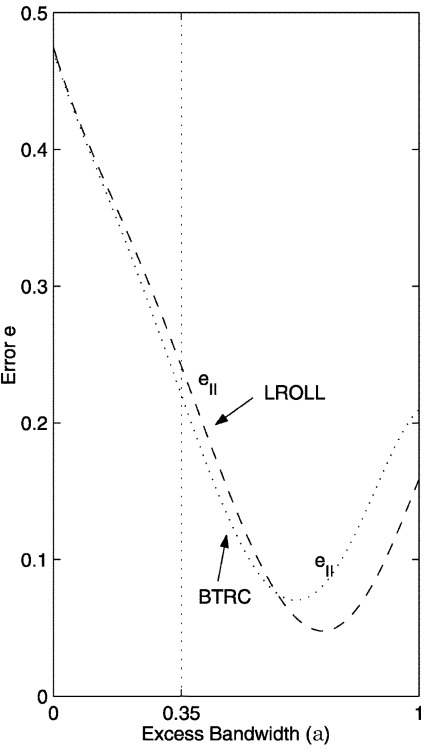

(b)
Fig. 2. Error plots of various pulses.

AWGN channel, the latter approximating a saturated Class C power amplifier.

For the different pulse shapes, we compared the root-meansquare error with respect to the $\mathrm{N} 1$ and $\mathrm{N} 2$ criteria as a function of the excess bandwidth in Fig. 2. The RC2 and TRC are the only pulses having errors in $e_{I}$, with the TRC having less error. In terms of the $e_{I I}$ criterion, the the RC has the most error, the 


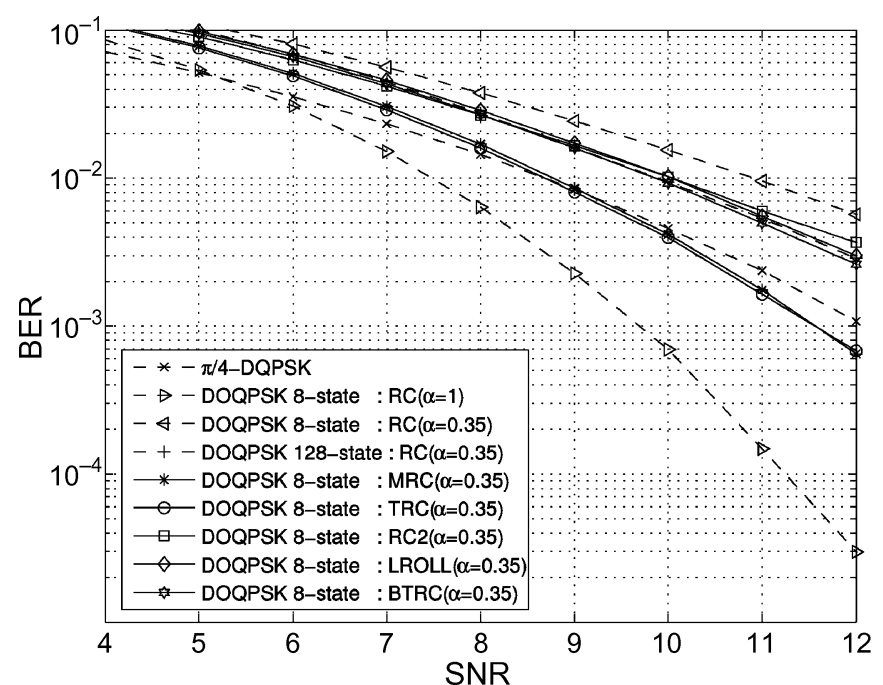

(a)

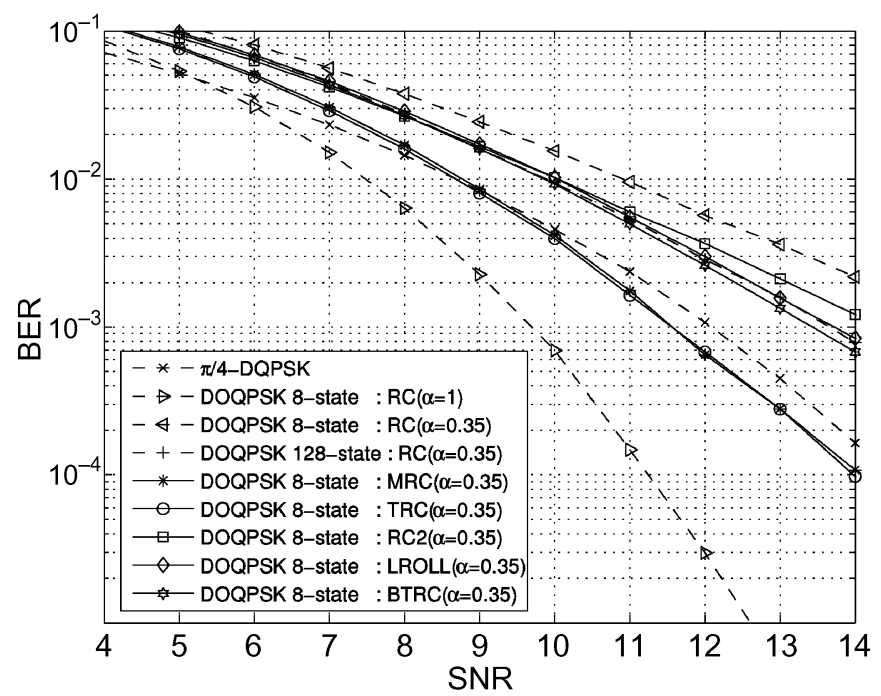

(b)

Fig. 3. Performance comparison of DOQPSK with proposed pulses (MRC, TRC, RC2, LROLL, BTRC). (a) AWGN channel for $\alpha=0.35$. (b) Hard-limited AWGN channel for $\alpha=0.35$.

TRC has the least error, and the MRC error is in-between, at any excess bandwidth. The two continuous pulses, LROLL and BTRC, yield less error in $e_{I I}$ than the RC, but more error than the other pulses at $\alpha=0.35$.

The bit-error rate (BER) of the DOQPSK system is shown for the AWGN channel in Fig. 3(a) and for the hard-limited AWGN channel in Fig. 3(b), using different pulse shapes at a roll-off factor of $\alpha=0.35$. Shown for reference are the $\pi / 4$-DQPSK system with an RC pulse having $\alpha=0.35$, the DOQPSK system with $\alpha=1$, and the 128-state DOQPSK [2], [3] receiver. The $\alpha=1$ DOQPSK receiver represents the best achievable performance, and the $\alpha=0.35$ DOQPSK RC pulse has the worst performance. Of the candidate pause shapes, MRC and TRC pulses had the best performance: $0.1 \mathrm{~dB}$ worse than the $\pi / 4$-DQPSK system in the AWGN channel, and $0.5 \mathrm{~dB}$ better in the hard-limited AWGN channel at a nominal BER of $10^{-3}$. The 128-state DOQPSK system in [2] and [3] had worse performance than the MRC and TRC pulses, followed by the BTRC, LROLL, and $\mathrm{RC} 2$ pulses, with the spread in performance being larger in the hard-limited channel. The relatively poor performance of the RC2 pulse stems from correlation in the noise samples that results when $\mathrm{N} 1$ is not satisfied.

\section{SUMmary}

Pulse shaping is an effective means to improve performance in DOQPSK system with constrained bandwidth. In both the AWGN and hard-limited AWGN channels, the MRC and TRC pulses with an 8 -state trellis outperformed the more complex 128 -state solution in [2] and [3]. These pulses show similar performance to the $\pi / 4$-DQPSK system in the AWGN channel, and a $0.5 \mathrm{~dB}$ performance improvement over the $\pi / 4$-DQPSK system in the hard-limited AWGN channel.

\section{REFERENCES}

[1] K. Defly, M. Lecours, and N. Boutin, "Differential detection of the OQPSK signal: Coding and decoding," in Proc. IEEE Int. Conf. Commun., Boston, MA, 1989, pp. 1660-1664.

[2] S. Hischke and J. Habermann, "A new differential demodulator for offset-QPSK," Int. J. Electron. Commun., vol. 52, no. 6, pp. 375-383, Dec. 1998.

[3] C. Guenther and J. Habermann, "DOQPSK-Differential demodulation of filtered offset QPSK," in Proc. IEEE Veh. Technol. Conf., 1994, pp. $1542-1546$.

[4] M. Simon, "Multiple-bit differential detection of offset QPSK," IEEE Trans. Commun., vol. 51, no. 9, pp. 1004-1011, Sep. 2003.

[5] L. L. R. Schober and I. Ho, "Enhanced multiple-bit differential detection of DOQPSK," IEEE Trans. Commun., vol. 53, no. 9, pp. 1490-1497, Sep. 2005.

[6] E. S. Sousa and S. Pasupathy, "Pulse shape design for teletext data transmission," IEEE Trans. Commun., vol. COM-31, no. 7, pp. 871-878, Jul. 1983.

[7] O. de Koe and P. der Wurf, "On some extensions of Nyquist's telegraph transmission theory," Proc. IEEE, vol. 57, no. 4, pp. 701-702, Apr. 1969.

[8] A. C. C. Lam, "Differential receiver design for the offset-QPSK modulation scheme," Master's thesis, Univ. Toronto, Toronto, ON, Canada, Apr. 2004.

[9] N. C. Beaulieu, C. C. Tan, and M. O. Damen, "A better than Nyquist pulse," IEEE Commun. Lett., vol. 5, no. 9, pp. 367-368, Sep. 2001. 